\title{
The road to Cybathlon 2016 - Functional electrical stimulation cycling Team IRPT/SPZ
}

\author{
Marco Laubacher (1,2), Efe Anil Aksöz (1,2), Ines Bersch (3), Kenneth James Hunt (1)
}

(1) Institute for Rehabilitation and Performance Technology, Division of Mechanical Engineering, Department of Engineering and Information Technology, Bern University of Applied Sciences, Burgdorf, Switzerland; (2) Sensory Motor Systems Lab, ETH Zurich, Zürich, Switzerland; (3) Swiss Paraplegic Centre Nottwil, Switzerland

This article is distributed under the terms of the Creative Commons Attribution Noncommercial License (CC BY-NC 4.0) which permits any noncommercial use, distribution, and reproduction in any medium, provided the original author $(s)$ and source are credited.

\begin{abstract} the competition. Cybathlon
\end{abstract}

Functional electrical stimulation (FES) provides a good possibility to activate paralysed muscles and it has been shown to elicit substantial physiological and health benefits. For successful application of FES, a perfect symbiosis of the bike and the pilot has to be achieved. The road to the Cybathlon 2016 describes the different pieces needed for FES cycling in spinal cord injury. The systematic optimisation of the stimulation parameters and the Cybatrike, and sophisticated training contributed to the team's success as the fastest surface-electrode team in

Key Words: Functional electrical stimulation, recumbent cycling, spinal cord injury,

Eur J Transl Myol 27 (4): 259-264
Functional electrical stimulation (FES) provides a good possibility to activate paralysed muscles and it has been shown to elicit substantial physiological and health benefits. For successful application of FES, a perfect symbiosis of the bike and the pilot has to be achieved. The road to the Cybathlon 2016 describes the different pieces needed for FES cycling in spinal cord injury. The Systematic optimisation of the Cybatrike and the stimulation parameters together with sophisticated trainings contributed to the team's success as the fastest surface-electrode team in the competition.

\section{Material and Methods}

\section{Pilot details}

The main inclusion and exclusion criteria were chosen according the Cybathlon 2016 rules. Additionally, our pilot was required to be willing and motivated to train alone during the preparation time. This included three to four regular FES sessions every week and some additional, intense training days. The recruitment started around 24 months in advance and turned out to be more difficult than expected. Despite the good cooperation with the spinal cord injury (SCI) centre in Nottwil, no Swiss pilot could be found. A collaboration with the ENS Lyon, which started after the Cybathlon rehearsal in 2015, led to the contact with the actual pilot from France. Our pilot was 37 years old, $182 \mathrm{~cm}$ tall and weighs $74 \mathrm{~kg}$. He is tetraplegic on the level C7 - C8 due to a road accident in July 2012 and he has an ASIA impairment scale score of A (complete loss of motor and sensory function).

\section{Tricycle information}

We used a VTX tricycle from Inspired Cycling Engineering (ICE, http://www.icetrikes.co) as the basis of our Cybatrike (Tab. 1). This trike has two 20" wheels at the front and a 28 " wheel at the back. The frame, seat and wheels were not modified. The weight of the trike out of the box was $14 \mathrm{~kg}$ and the fully equipped bike with stimulator, controller, electrodes, cables, sensors and battery was $19.2 \mathrm{~kg}$. Derailleur and shifter were replaced with an electric gear change mechanism: Shimano Ultegra DI (Shimano Inc., Japan). The crank set was replaced with an asymmetric chain ring (Osymetric BCD130mm - 42, Osymetric, France) and a $165 \mathrm{~mm}$ road racing crank (Sugino RD II Messenger JIS, Sugino, Italy), which minimized the Q-factor for more ergonomic pedalling. This was necessary due to a sensor placed on the bottom bracket. The sensor was used to detect the pedal angle and to set the timing for the stimulation. Since our pilot has reduced hand function, a double barrelled brake lever (Double Barrel, Problemsolvers Inc., USA) was mounted, which allows the control of two brake callipers with one lever. 
Table 1. Cybatrike specifications

\begin{tabular}{ll}
\hline Overall width: & $750 \mathrm{~mm}$ \\
Overall height: & $690 \mathrm{~mm}$ \\
Overall length: & $2050 \mathrm{~mm}$ \\
Turning circle: & $7.4 \mathrm{~m}$ \\
Front wheel size: & $20^{\prime \prime}$ \\
Rear wheel size: & $28^{\prime \prime}$ \\
Seat: & Carbon \\
Brake Caliper: & Avid BB7 disc brakes \\
Brake Lever: & Problemsolver \\
& Barrel Double \\
Gear System: & Shimano Ultegra DI, 10 \\
& speed (12 - 34) \\
Crank System: & Sugino RD II, 165 mm with \\
& Asymmetric chain ring 42 \\
Rims: & Alexrims DA22 \\
Tyres: & Schwalbe Kojak \\
Weight: & 19.2 kg \\
\end{tabular}

The pilot was almost in a supine position (see Fig. 1). Measured from a horizontal line, the upper body angle was around $35 \mathrm{deg}$. Leg/hip angle measured from the same horizontal line was around $15 \mathrm{deg}$ upwards. At the maximal pedal distance, the knee angle was around 30 $35 \mathrm{deg}$ (straight leg means zero deg), which is in the recommended range for maximal pedal force generation. ${ }^{1}$

Professional custom foot/ankle orthoses (Orthoteam AG, Switzerland) were made to keep hip, knee and ankle joints in one line during the stimulated cycling and to opti $\neg$ mize force transmission to the pedals.

\section{Electrical stimulation during the race}

The coordination of the different muscle groups to achieve a good cycling movement seemed at first to be very difficult. There are four main muscle groups involved in generating a smooth pedal movement: the m. quadriceps, the hamstrings, the gluteal muscles and the $\mathrm{m}$. triceps surae. ${ }^{2,3}$ The low upper body position reduced the influence of the $\mathrm{m}$. gluteus, which therefore was not stimulated. The ankle joint was fixed with the custom foot orthosis, so that no stimulation was needed for the $\mathrm{m}$. triceps surae. The $\mathrm{m}$. hamstrings produce less power than the $\mathrm{m}$. quadriceps but they help to overcome the dead pedal position. To investigate the influence of the hamstring muscles we conducted a $20 \mathrm{~m}$ speed test with and without activated hamstrings (Tab. 2). In addition to the measured time, it was important to see how smoothly the activated muscles worked together. Thus, inter-muscular coordination was our second decisive factor. The difference in time was very small but with activated hamstrings, the pedal stroke was not as smooth as with quadriceps activation only. We observed co-contractions in knee extensor muscles during the stimulation of the hamstring muscles, which inhibited a smooth pedal stroke. Neither reducing pulse intensity nor replacing the electrodes improved this coordination. With this subject, we therefore finally decided to stimulate only the $\mathrm{m}$. quadriceps, since this setup caused a more ergonomic and more natural cycling movement. Due to the supine pilot position, gravity helped to overcome the dead pedal position (see Fig. 1). For the Cybathlon race, we used a RehaStim I stimulator (Hasomed GmbH, Germany) with eight channels. Two electrodes $(4.5$ x $9 \mathrm{~cm}$; Pals, Axelgaard Manufacturing Co, USA) were placed on each m. vastus lateralis and $\mathrm{m}$. vastus medialis, respectively, and two electrodes $(2.5 \times 2.5 \mathrm{~cm}$; Pals, Axelgaard Manufacturing Co, USA) were transversely applied to stimulate the $\mathrm{m}$. rectus femoris. Two channels stayed unused. Stimulation frequency was $35 \mathrm{~Hz}$, amplitude was 120 $\mathrm{mA}$ for $\mathrm{m}$. vastus medialis and $\mathrm{m}$. vastus lateralis, respectively, and $80 \mathrm{~mA}$ for the $\mathrm{m}$. rectus femoris. We used simple bi-phasic pulse signals with constant amplitude and varying pulse width. The pulse width was changed via a throttle controlled by the pilot and went up to $500 \mu \mathrm{s}$. No direct visual feedback was available on the Cybatrike for the stimulation intensity. We therefore marked the $100 \%$, the $75 \%$ and the $50 \%$ throttle positions, with the corresponding stimulation intensities.

\section{Training information and preparation}

The first stimulation series started one year before Cybathlon and regular training sessions started 10 months before the race. We had two training camps at

Table 2. Time is the duration in seconds for a $20 \mathrm{~m}$ indoor test run with three different electrode setups. The basic setup consisted of two electrodes on m. quadriceps. All muscles were stimulated with $35 \mathrm{~Hz}, 80 \mathrm{~mA}$ and a maximal pulse width of $500 \mu \mathrm{s}$.

\begin{tabular}{llllll}
\hline Time [s] & Run 1 & Run 2 & Run 3 & Run 4 & Run 5 \\
Activated muscles & & & & & \\
With hamstrings & 7.8 & 9.7 & 10.3 & & \\
Without hamstrings & 8.7 & 9.7 & 10 & 9.4 & 9.9 \\
3 electrodes on m. quadriceps & 8.8 & 9.6 & 9.7 & 9.4 & \\
\hline \hline
\end{tabular}


a)

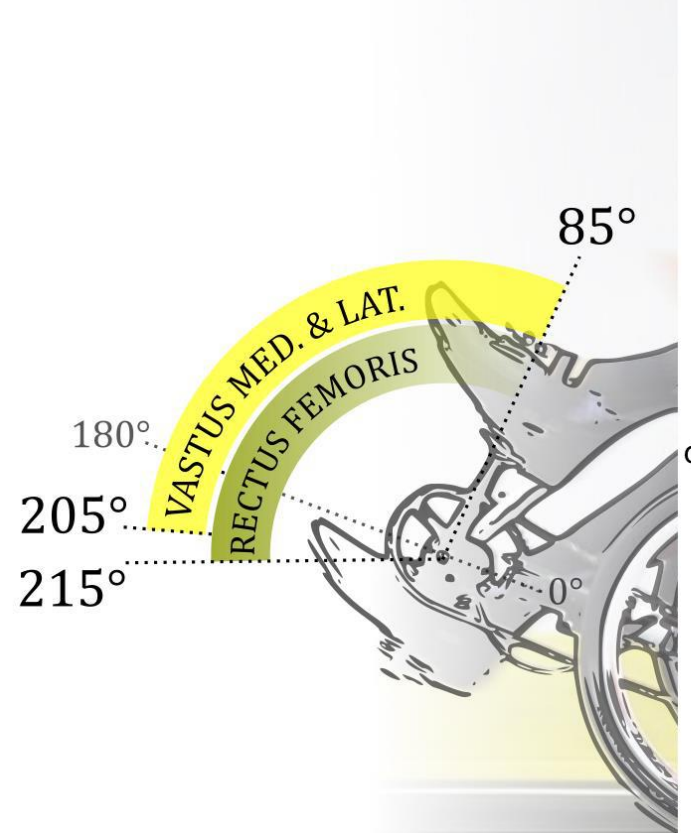

b)
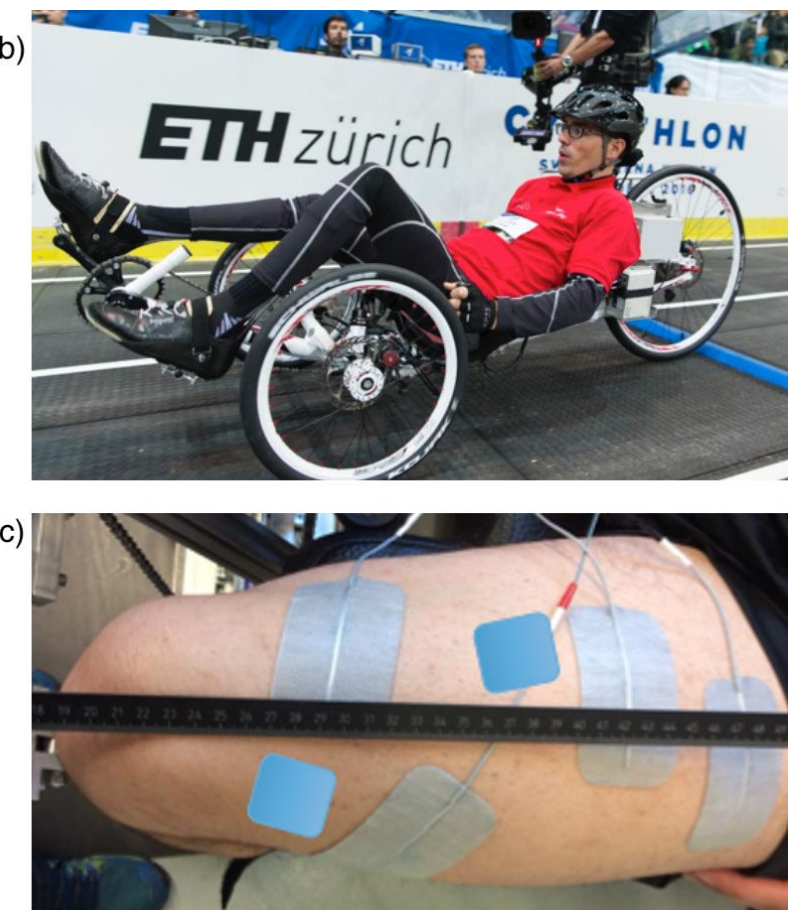

Fig 1. (a) Activation angles of the $\mathrm{m}$. vastus lateralis, the $\mathrm{m}$. vastus medialis and the $\mathrm{m}$. rectus femoris. Zero deg is in line with the frame. (b) The Cybatrike of the team IRPT/SPZ and the pilot on the recumbent bike. (c) The electrode setup used for the race.

Nottwil's outdoor sports facility lasting several days to tune the setup and to adjust the device perfectly to our pilot. Muscles were stimulated five to six times per week at least one hour per day. Two to three FES cycling sessions per week were planned and were mainly conducted on a stationary device (RT 300, RTI Inc., USA) in an upright sitting position. Comparable to conventional resistance training, those sessions were varied in volume and intensity and continuously adapted to the training status. We combined interval and constant training sessions, whereby stimulation time was between 60 and 90 minutes per session. ${ }^{4}$ The training load was dependent on the applied stimulation intensity and on the training duration. A typical constant training session started with a target power output of $8 \mathrm{~W}$ for 20 minutes, then $12 \mathrm{~W}$ for 10 minutes and then again $8 \mathrm{~W}$ for 30 minutes. In contrast, an interval session started with $8 \mathrm{~W}$ for 10 minutes and then consisted of three high intensity phases with steps up to $18 \mathrm{~W}$ for 2 minutes. Between each step there was a low intensity phase at $8 \mathrm{~W}$ for 2 minutes. Stimulation was adapted and controlled in a closed-loop system according to target power output and a target pedalling cadence of $50 \mathrm{rpm}$. The daily home training sessions without the bike were conducted at low intensities and were used as basic activation and muscle conditioning. ${ }^{5-7}$ Electrodes were applied on the quadriceps $\mathrm{m}$., hamstrings $\mathrm{m}$. and glutei $\mathrm{m}$. for most of the training sessions. During the preparation time, electrodes were intentionally placed on different areas. We expected to train more and different muscle fibres and finally to get a better overall activation, even if the actual training power output seemed to be low. Weekly stretching of the trained muscles helped to release muscle tension and kept the muscles elastic., 8

\section{Stimulation strategies}

Next to a good bike and stimulation setup, it was important to approach the race with a distinct strategy. Muscle fatigue is known to be a major limitating factor. ${ }^{10}$ Since pulse width was the only parameter to be changed during the race, we had to decide how to use the throttle to be as fast as possible on the $750 \mathrm{~m}$ track. We tested the following five different conditions during the second training camp two weeks before the competition (Tab. 3):

[S1] All-out strategy. Go as fast as possible, use highest pulse width from the start.

[S2] Stay constant. Start with a slightly lower pulse width and try to be as con $\neg$ stant as possible over the whole dis $\neg$ tance. Intuitively controlled by the athlete.

[S3] Increase. Start with low pulse width and steadily increase it. Focus on the throttle, and increase the pulse width.

[S4] Fatigued constant. Same as second strategy but with pre-fatigued muscles.

[S5] High amplitude, constant: Stay constant but this time the amplitude was in $\neg$ creased to $130 \mathrm{~mA}$.

Based on the split times of those training runs, athlete feedback and observations, we decided to go with a mixture of S2 and S3. The pedal frequency is a critical 
Table 3. Speed for $705 \mathrm{~m}$ outdoor test runs. For each run a different strategy was applied. The Amplitude was the same for each electrode.

\begin{tabular}{|c|c|c|c|c|c|c|c|c|}
\hline \multirow{2}{*}{\multicolumn{2}{|c|}{$\begin{array}{l}\text { Strategy } \\
\qquad \text { Amplitude }\end{array}$}} & & Split 1 & Split 2 & Split 3 & Split 4 & Split 5 & Total \\
\hline & & & & & & & & \\
\hline S1 & $80 \mathrm{~mA}$ & Speed $[\mathrm{km} / \mathrm{h}]$ : & 11.52 & 10.24 & 8.23 & 6.61 & 4.59 & 8.24 \\
\hline $\mathrm{S} 2$ & $120 \mathrm{~mA}$ & Speed $[\mathrm{km} / \mathrm{h}]$ : & 12.52 & 9.16 & 9.29 & 9.00 & 8.74 & 9.74 \\
\hline S3 & $120 \mathrm{~mA}$ & Speed $[\mathrm{km} / \mathrm{h}]$ : & 12.00 & 11.35 & 9.60 & 8.85 & 6.85 & 9.73 \\
\hline S4 & $120 \mathrm{~mA}$ & Speed $[\mathrm{km} / \mathrm{h}]$ : & 9.60 & 7.68 & 7.20 & 7.05 & 7.12 & 7.73 \\
\hline S5 & $130 \mathrm{~mA}$ & Speed $[\mathrm{km} / \mathrm{h}]$ : & 13.09 & 12.73 & 7.02 & & & 10.95 \\
\hline
\end{tabular}

factor for high power output, ${ }^{11}$ and pedalling around 50 rpm delivered the best performance during the rehearsal. The aim of that progressive strategy was to use around $75-80 \%$ of the maximum throttle in the first round and then steadily increase the pulse width to maintain the pedal frequency and speed. Since pulse width can not be increased indefinitely and fatigue increases during the race, shifting to lower gears is essential to maintain the optimal pedalling frequency.

\section{Results}

\section{Performance obtained at Cybathlon 2016}

Immediate preparation for the race included unloaded FES-Cycling and stretching of the lower leg muscles. The stretching was focused on the hamstrings and the m. glutei. Based on our rehearsal runs, we were prepared for a progressive strategy. One unknown and important factor was the resistance of the ground and which gear would be necessary for $50 \mathrm{rpm}$. We started with the 3rd gear (26 teeth) and tried to shift up to 4th (23 teeth) $10-20 \mathrm{~m}$ after the start. In the qualification run our pilot increased the throttle somewhat more than intended and the initial stimulation intensity was therefore too high. The muscles fatigued and the resistance was too high to maintain a constant pedal frequency (Fig 2a). Shifting to a lower gear didn't help and speed decreased due to the increasing fatigue. The mean velocity in the qualification round was $8.3 \mathrm{~km} / \mathrm{h}$. In contrast, in the final round our pilot started with the second gear (32 teeth) and managed to apply the strategy almost perfectly. Stimulation intensity and gear were chosen and controlled very well and he therefore managed to keep the pedal frequency almost constant (Fig 2b). The final run was more than $25 \%$ faster with a mean velocity of $11.3 \mathrm{~km} / \mathrm{h}$. In fact, this final round performance was the fastest overall performance of any
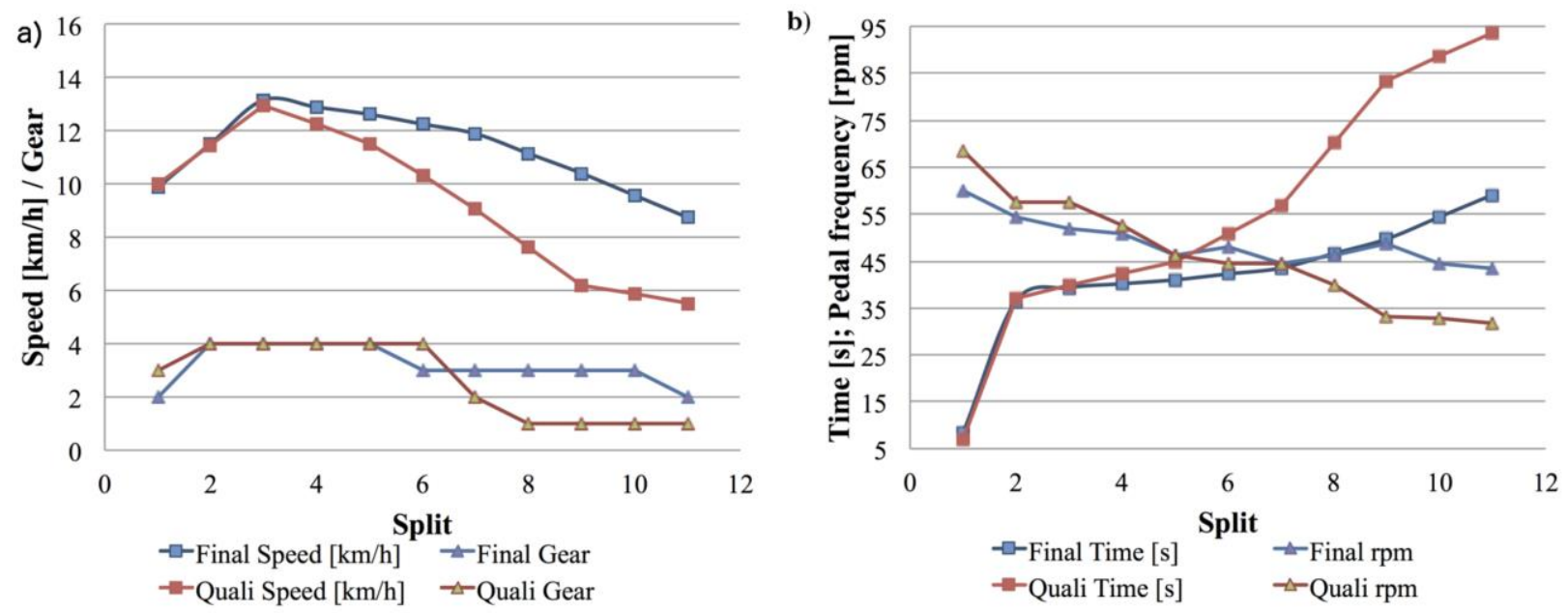

Fig 2. (a) The speed in $\mathrm{km} / \mathrm{h}$ and the selected gear for the qualification run in the morning and the final run in the afternoon. Gear means chain position on the rear cassette (range 11 - 34 dents, whereby 1 means chain on the biggest $\operatorname{cog}$ ). (b) The time in seconds and the pedalling cadence [rpm] for both runs. Both figures show the split times on the $\mathrm{x}$-axis. Split one was $19 \mathrm{~m}$ and all other splits were $73.5 \mathrm{~m}$, in total $750 \mathrm{~m}$. 


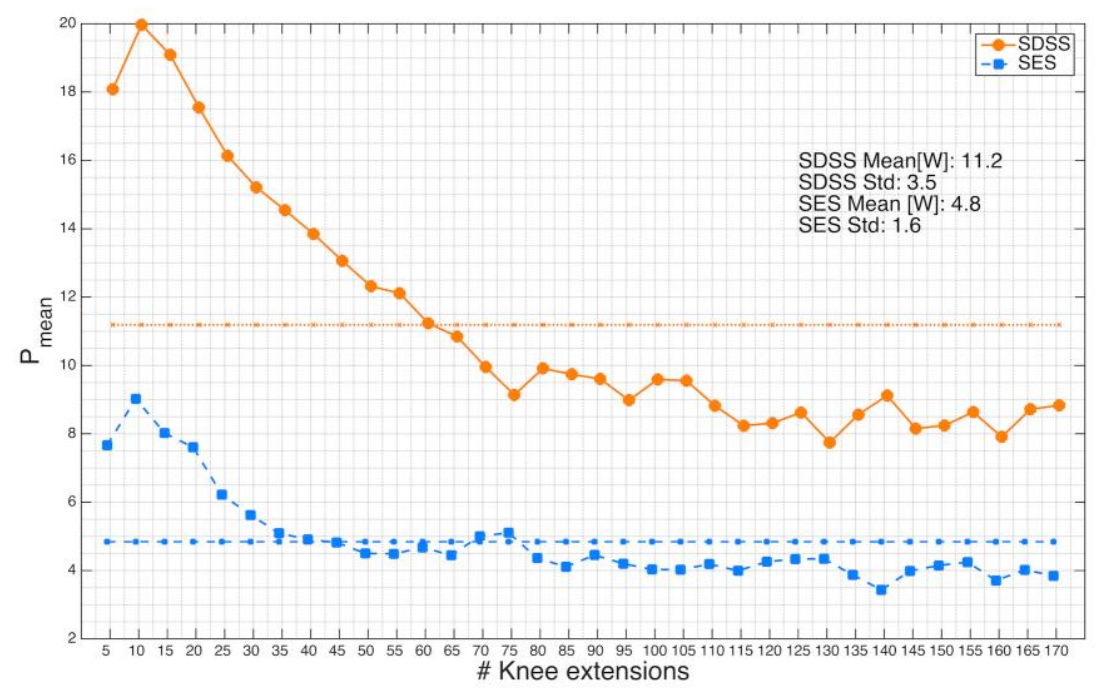

Fig 3. Mean power output (Pmean) during a 6-minute stimulated knee extension task. The quadriceps were stimulated during knee extension, simulating the knee joint movement during recumbent cycling. The lines represent the means of the right and the left legs combined. The orange line shows the power output with a spatial and sequential distributed electrode setup (SDSS) and the blue line is the power output with a conventional single electrode setup (SES); the latter was used during the Cybathlon race. Stimulation parameters were 4 x $8.75 \mathrm{~Hz}, 40 \mathrm{~mA}$ and $140 \mu$ s for the SDSS setup and $35 \mathrm{~Hz}, 40 \mathrm{~mA}$ and $155 \mu \mathrm{s}$ for the standard setup.

pilot using surface electrodes at the Cybathlon 2016 FES bike race. However, due to the slower time in the qualifying race, our pilot was in the B-final and therefore won the bronze medal.

\section{Discussion}

The application of functional electrical stimulation to mobile cycling for spinal cord injured people is a challenge and it is not only stimulation that matters, it is about a perfect symbiosis of the bike and the pilot. For this reason we spent considerable time and effort in tuning the bike and stimulation strategy to the physiological and neurological characteristics and capabilities of our particular pilot. Ergonomic pedalling and efficient force transmission are as important as the stimulation strategy and power generated. The main difficulty is to control the stimulation intensity during cycling, since the pilot has no direct feedback about muscle status. A pedal force or power meter including gear selection would give important feedback to the pilot and help him to control the stimulation intensity during the cycling. During the training period, the handling of the stimulator, the electrodes and finally the bike is a further challenge. Even with a familiar setup, it takes about 30 minutes of preparation to properly set up the elec-trodes, transfer to the bike, fix the legs to the orthoses and connect the cables. At least one additional person was needed to place the electrodes and to help with the transfer to the bike. Alternatively, surface electrodes, already implemented in a wearable garment would facilitate its application and save a lot of time, which finally would help with training on a daily basis. During training, stimulating the target mus $\neg$ cles while pedalling was essential for building up the right structures. Training with the RT 300 was a little less time-consuming and a good possibility to train on a controlled basis, by increasing or decreas-ing stimulation according a target power when needed. In contrast to the Cybatrike, the RT 300 increased the stimulation intensity by changing the amplitude. This might by a good option for a diversified training, but for the next preparation period, more training time should be spent on the actual race bike. For future events, stochastic inter-pulse interval and a spatial and sequential distributed electrode setup (SDSS) should be used as this strategies has been shown to improve power and fatigue characteristics. ${ }^{12-14} \mathrm{~A}$ test measurement with our pilot according a protocol published by Laubacher et al. ${ }^{12,15}$ showed an increased power output for the SDSS setup during a six minute knee extension task (Fig. 3). But that setup needs more channels, thus another stimulator together with a demultiplexor or a coupling of at least two stimulators is needed. ${ }^{16}$ The latter brings more weight and requires more space. Thus, only a device which allows the use of more than 24 electrodes would be an option.

\section{List of acronyms}

AIS A - American spinal injury association Impairment Scale score A means no sensory or motor function is preserved in sacral segments S4 - S5

ICE - Inspired Cycling Engineering 
Q-Factor - Horizontal distance between the two pedal attachment points, measured parallel to the bottom bracket.

\section{Author's contributions}

ML is the principle author and responsible for the clinical protocol, pilot training and the bike setup. EAA was responsible for the technical integration and software design of this project. IB was involved in the clinical protocol, pilot training and follow up and $\mathrm{KJH}$ was the leader of the team. All the authors read and approved the final version of the manuscript.

\section{Acknowledgments}

The authors gratefully acknowledge the Swiss Paraplegic Centre and the Swiss Medical Centre in Nottwil for supporting this project and for providing use of their training facilities. This work was supported in part by the Swiss National Science Foundation (SNF Grant No. 320030_150128/1).

\section{Conflict of Interest}

The authors declare the absence of any commercial or financial relationships or potential conflicts of interest.

\section{Ethical Publication Statement}

We confirm that we have read the Journal's position on issues involved in ethical publication and affirm that this report is consistent with those guidelines.

\section{Corresponding Author}

Marco Laubacher, Pestalozzistrasse 20, 3400 Burgdorf, Switzerland. E-mail: marco.laubacher@bfh.ch

\section{E-mails of co-authors}

Efe Anil Aksöz: efeanil.aksoez@bfh.ch

Ines Bersch: ines.bersch@paraplegie.ch

Kenneth James Hunt: kenneth.hunt@bfh.ch

\section{References}

1. Kato M, Tsutsumi $\mathrm{T}$, Yamaguchi $\mathrm{T}$, et al. Characteristics of maximum performance of pedaling exercise in recumbent and supine positions. J Sports Sci Med 2011;10:491-7.

2. Hakansson NA, Hull ML. Functional roles of the leg muscles when pedaling in the recumbent versus the upright position. J Biomech Eng 2005;127:301-10.

3. Szecsi J, Straube A, Fornusek C. Leg general muscle moment and power patterns in able-bodied subjects during recumbent cycle ergometry with ankle immobilization. Med Eng Phys 2014;36:1421-7.

4. ACoS Medicine, ACSM's Guidelines for Exercise Testing and Prescription: Wolters Kluwer/Lippincott Williams \& Wilkins Health, 2013.
5. Gorgey S, Mahoney E, Kendall T, Dudley GA. Effects of neuromuscular electrical stimulation parameters on specific tension. Eur J Appl Physiol 2006;97:737-44.

6. Mahoney ET, Bickel CS, Elder C, et al. Changes in skeletal muscle size and glucose tolerance with electrically stimulated resistance training in subjects with chronic spinal cord injury. Arch Phys Med Rehabil 2005;86:1502-4.

7. WadeR C, Gorgey AS. Skeletal muscle conditioning may be an effective rehabilitation intervention preceding functional electrical stimulation cycling. Neural Regen Res 2016;11:1232-3.

8. Masugi Y, Obata H, Inoue D, et al. Neural effects of muscle stretching on the spinal reflexes in multiple lower-limb muscles. PLoS One 2017;12:e0180275.

9. Apostolopoulos N, Metsios GS, Flouris AD, et al. The relevance of stretch intensity and position - a systematic review. Front Psychol 2015;6:1128.

10. Hunt KJ, Fang J, Saengsuwan J, et al. On the efficiency of FES cycling: A framework and systematic review. Technol Health Care 2012;20:395-422.

11. Fornusek C, DavisGM, Russold MF. Pilot study of the effect of low-cadence functional electrical stimulation cycling after spinal cord injury on thigh girth and strength. Arch Phys Med Rehabil 2013;94:990-3.

12. Laubacher M, Aksoz AE, Riener R, et al. Power output and fatigue properties using spatially distributed sequential stimulation in a dynamic knee extension task. Eur J Appl Physiol 2017 Jul 3. doi: 10.1007/s00421-017-3675-0.

13. Nguyen R, Masani K, Micera S, et al. Spatially distributed sequential stimulation reduces fatigue in paralyzed triceps surae muscles: a case study. Artif Organs 2011;35:1174-80.

14. AksozEA, Laubacher M, Binder-Macleod S, Hunt KJ. Effect of Stochastic Modulation of Inter-Pulse Interval During Stimulated Isokinetic Leg Extension. Eur J Transl Myol 2016;26:6160.

15. Laubacher M, Aksoz EA, Binder-Macleod S, KJ Hunt. Comparison of Proximally Versus Distally Placed Spatially Distributed Sequential Stimulation Electrodes in a Dynamic Knee Extension Task. Eur J Transl Myol 2016;26:6016.

16. ValtinM, Kociemba K, Behling $\mathrm{C}$, et al. RehaMovePro: A Versatile Mobile Stimulation System for Transcutaneous FES Applications. Eur J Transl Myol 2016;26:6076.

17. Azevedo Coste C, Bergeron V, Berkelman R, et al. Comparison of strategies and performance of functional electrical stimulation cycling in spinal cord injury pilots for competition in the first ever Cybathlon. Eur J Transl Myol 2017;27:251-4. 